\title{
DIGESTIBILIDADE DO AMIDO E DISPONIBILIDADE DE Ca e P EM ALIMENTOS ENERGÉTICOS EXTRUSADOS PARA A TILÁPIA DO NILO (Oreochromis niloticus)
}

\author{
Igo Gomes Guimaraes ${ }^{1}$, Luiz Edivaldo Pezzato ${ }^{2}$, Margarida Maria Barros ${ }^{2}$, Leonardo \\ TACHIBANA $^{3}$, ROSANGELA DO NASCIMENTO FERNANDES ${ }^{4}$ \\ ${ }^{1}$ - Professor Doutor, Universidade Federal de Goiás, Campus Jataí,GO - igoguimaraes@gmail.com \\ 2 - Professores Doutores Universidade Estadual Júlio de Mesquita Filho - Botucatu, SP \\ 3 - Pesquisador científico da Agência Paulista de Tecnologia dos Agronegócios - Instituto de Pesca - São Paulo, SP \\ ${ }^{4}$ - Doutoranda do Centro de Aquicultura da Unesp - Caunesp - Jaboticabal, SP
}

\section{RESUMO}

O coeficiente de digestibilidade aparente (CDA) do amido e a disponibilidade aparente do cálcio $(\mathrm{Ca})$ e fósforo $(\mathrm{P})$ de cinco alimentos energéticos (milho, farelo de trigo, quirera de arroz, farelo de arroz e sorgo) foram avaliados para a tilápia do Nilo. O óxido de crômio-III foi utilizado como indicador inerte das rações. Apresentaram os maiores $\mathrm{CDA}$ do $\mathrm{Ca}$ e $\mathrm{P}$, respectivamente, a quirera de arroz (43,03 e 64,79\%), o sorgo $(39,89$ e $58,09 \%)$ e o milho $(22,18$ e $19,48 \%)$, enquanto o farelo de arroz $(-31,49$ e $3,25 \%)$ e o farelo de trigo $(5,80$ e $1,18 \%)$ apresentaram os menores valores. A digestibilidade do amido apresentou-se entre 99,45 e $95,59 \%$ entre os alimentos energéticos avaliados. $\mathrm{O}$ alto CDA do amido observado neste estudo confirma a grande capacidade da tilápia do Nilo de utilizar carboidratos nas formas mais complexas.

PALAVRAS-CHAVE: carboidratos; CDA; extrusão; minerais; tilápia.

\section{STARCH DIGESTIBILITY AND Ca AND P AVAILABILITY IN CEREAL GRAIN PRODUCTS AND BY-PRODUCTS IN EXTRUDED DIETS FOR NILE TILAPIA (Oreochromis niloticus)}

\section{ABSTRACT}

Apparent digestibility coefficients (ADC) of starch and calcium (Ca) and phosphorus (P) apparent availability were evaluated in five cereal grain products and byproducts (corn, wheat meal, rice grain, rice bran and sorghum) for Nile tilapia. Chromic oxide was used as an external digestibility marker. The highest $\mathrm{Ca}$ and $\mathrm{P}$ apparent availability, respectively, were obtained for rice grain (43.03 and 64.79\%), sorghum (39.89 and 58.09\%) and corn (22.18 and $19.48 \%$ ), while rice bran (-31.49 and $3.25 \%$ ) and wheat meal (5.80 and $1.18 \%$ ) showed the lowest values. Starch digestibility varied between 99.45 and $95.59 \%$ among the evaluated ingredients. This high ADC of starch observed in this study confirms that Nile tilapia is able to efficiently digest and utilize complex carbohydrates.

KEYWORDS: ADC; carbohydrates; extrusion; mineral; tilapia. 


\section{INTRODUÇÃO}

A aquicultura intensiva contemporânea requer a utilização de uma alimentação balanceada, à base de rações formuladas com os mais diversos ingredientes e processos de elaboração para um melhor aproveitamento pelos peixes. O impacto da piscicultura na poluição dos corpos d'água tem ganhado importância mundial visto $\mathrm{o}$ atual crescimento desta atividade. Com isso, vários autores têm salientado a importância do conhecimento da disponibilidade dos nutrientes nas rações e do desenvolvimento de dietas que reduzam a carga poluente gerada pela aquicultura, principalmente em relação à emissão de fósforo $(\mathrm{P})$ e outros minerais no ambiente, fator determinante para o processo de eutrofização do meio (CHO \& BUREAU, 2001; MACMILLAN et al., 2003; TACON \& FORSTER, 2003; HUA et al., 2005; SUGIURA et al, 2006).

O coeficiente de digestibilidade aparente $(\mathrm{CDa})$ é uma das formas mais práticas para se estimar a disponibilidade dos nutrientes nos alimentos e pode ser utilizado na escolha de alimentos que melhorem o valor nutritivo e o custo das rações (FAGBENRO, 1998). Desta maneira, grupos de pesquisa trabalham com diferentes espécies de peixes no sentido de obterem valores de nutrientes digestíveis para a indústria de alimentos.

Estudos demonstram que há significativa melhora na disponibilidade do fósforo em ingredientes de origem vegetal após o processo de extrusão, devido à redução entre $13-35 \%$ no conteúdo de fitato; entretanto, os resultados dos estudos são contraditórios (CHEFTEL, 1986).

A baixa digestibilidade de alguns alimentos de origem vegetal tem sido atribuída à quantidade e composição química do amido nesses alimentos. Adicionalmente, o hábito alimentar das espécies de peixes parece influenciar a utilização de carboidratos, sendo as espécies onívoras e herbívoras aquelas que aproveitam melhor essa fração dos alimentos quando comparadas às espécies de peixes carnívoras (MCGOOGAN \& REIGH, 1996). Desta forma, pesquisas anteriores têm demonstrado que a tilápia é capaz de utilizar mais eficientemente os carboidratos complexos que as formas mais simples (mono e dissacarídeos) (SHIAU, 1997). Apesar de o efeito do processamento da dieta sobre os coeficientes de digestibilidade dos alimentos já ter sido avaliado (TAKEUCHI et al., 1994; CHEN e HARDY, 2003), os estudos que determinam a digestibilidade do amido nos alimentos após processo de extrusão ainda são escassos.

Como se mostra clara a escassez de literatura relacionada ao efeito da extrusão sobre a disponibilidade dos nutrientes nos alimentos e rações para tilápia do Nilo, objetivou-se com este estudo avaliar a digestibilidade do amido e a disponibilidade de cálcio $(\mathrm{Ca})$ e fósforo $(\mathrm{P})$ de cinco alimentos energéticos extrusados para essa espécie.

\section{MATERIAL E MÉTODOS}

O experimento foi conduzido na Universidade Estadual Paulista (UNESP), no Laboratório de Nutrição de Organismos Aquáticos (AquaNutri) do Departamento de Melhoramento e Nutrição Animal (DMNA), da Faculdade de Medicina Veterinária e Zootecnia, Campus de Botucatu, SP.

Os alimentos avaliados no presente estudo foram o milho, farelo de trigo, quirera de arroz, farelo de arroz e sorgo. Todos os ingredientes foram moídos de forma a se apresentarem com diâmetro inferior a $500 \mu \mathrm{m}$. Em seguida, foram incorporados a uma dieta referência prática (Tabela 1 ) na relação de $6: 4(60,0 \%$ da dieta referência e $40,0 \%$ do alimentoteste).

Tabela 1. Composição da dieta referência

\begin{tabular}{lc}
\hline Ingredientes & $\mathrm{g} / 100 \mathrm{~g}$ da dieta \\
\hline Farelo de soja & 57,50 \\
Farinha de peixe & 5,50 \\
Quirera de arroz & 27,40 \\
Farelo de trigo $^{\text {Óleo de soja }}$ & 2,00 \\
Suplemento vitamínico $^{\mathrm{a}}$ & 0,50 \\
Suplemento mineral $^{\mathrm{b}}$ & 0,10 \\
Ácido ascórbico $^{\text {Oxido de crômio }}$ & 0,30 \\
L-Lisina $^{\text {DL-Metionina }}$ & 0,08 \\
Treonina $_{\text {Fosfato bicálcico }}$ & 0,10 \\
Calcário calcítico (se for) $_{\text {Cloreto de sódio }}$ & 0,70 \\
Antioxidante (BHT) $^{\mathrm{c}}$ & 0,45 \\
\hline
\end{tabular}

${ }^{a}$ Suplemento vitamínico, níveis de garantia por $\mathrm{kg}$ da dieta: vitamina $\mathrm{A}, 16060 \mathrm{UI}$; vitamina $\mathrm{D}_{3}, 4510 \mathrm{UI}$; vitamina $\mathrm{E}, 250$ UI; vitamina $K$, $30 \mathrm{mg}$; vitamina $B_{1}, 32 \mathrm{mg}$; vitamina $B_{2}, 32 \mathrm{mg}$; pantotenato de cálcio, $80 \mathrm{mg}$; niacina, $170 \mathrm{mg}$; biotina, $10 \mathrm{mg}$; ácido fólico, $10 \mathrm{mg}$; vitamina $\mathrm{B}_{12}, 32 \mu \mathrm{g}$; vitamina $\mathrm{B}_{6}, 32 \mathrm{mg}$.

b Suplemento mineral, níveis de garantia por $\mathrm{kg}$ da dieta: $\mathrm{Na}_{2} \mathrm{SeO}_{3}, 0,7 \mathrm{mg}$; $\mathrm{MnO}, 50 \mathrm{mg}$; $\mathrm{ZnO}, 150 \mathrm{mg}$; $\mathrm{FeSO}_{4}, 150 \mathrm{mg}$; $\mathrm{CuSO}_{4}, 20 \mathrm{mg} ; \mathrm{CoSO}_{4}, 0,5 \mathrm{mg} ; \mathrm{I}_{2} \mathrm{Ca}, 1 \mathrm{mg}$.

${ }^{\mathrm{c}}$ antioxidante Butil hidroxitolueno

O óxido de crômio-III foi usado como marcador externo na concentração de $0,1 \%$ da ração de acordo com BREMER NETO et al. (2005). As dietas foram mecanicamente homogeneizadas e água 
foi adicionada $(25,0 \%$ do peso natural) em misturador automático (Ação Científica ${ }^{\circledR}$ ) e então foram submetidas à extrusão (extrusora de rosca simples - Extrutec $($ ), de forma a se obterem péletes com aproximadamente $5,0 \mathrm{~mm}$ de diâmetro. As dietas foram secas em estufa com recirculação forçada de ar a $55,0^{\circ} \mathrm{C}$, por cerca de 12 horas, e armazenadas em freezer a $-18,0^{\circ} \mathrm{C}$.

Para determinação do coeficiente de digestibilidade aparente, utilizaram-se seis aquários de alimentação, com formato circular e capacidade de 250 litros de volume cada, onde as dietas experimentais foram distribuídas aleatoriamente, e outros três aquários para a coleta de fezes, com formato cônico e capacidade de 250 litros. Tanto os aquários de alimentação quanto os de coleta de fezes estavam ligados a um sistema de recirculação de água, com filtro físico e biológico e temperatura da água mantida por meio de termostato eletrônico e digital a $27,0 \pm 0,5^{\circ} \mathrm{C}$.

Os peixes (90 juvenis de tilápia do Nilo, com peso médio de $150 \pm 16,5 \mathrm{~g}$ ) foram alojados em seis tanques-rede de formato circular $(80,0 \mathrm{~cm}$ de diâmetro e $60,0 \mathrm{~cm}$ de altura) confeccionados em tela plástica (malha de $1,5 \mathrm{~cm}$ entre nós). Os tanques foram utilizados para abrigar os peixes e facilitar o manejo de alimentação e coleta de fezes. Os peixes foram mantidos nos aquários de alimentação onde receberam refeições à vontade.

Em seguida, foram transferidos para os aquários de coleta de fezes e permaneceram até a manhã do dia subsequente. Esses tanques, dotados de um sistema de coleta de fezes por gravidade, possibilitaram a obtenção do material para análise.

O período de coleta de fezes de cada um dos ingredientes foi de três dias para cada tanque, compondo material necessário para uma repetição, sendo as dietas distribuídas aleatoriamente a cada período de coleta, de forma a compor quatro repetições por alimento. As fezes foram secas em estufa de ventilação forçada a $52^{\circ} \mathrm{C}$ e moídas. As análises de amido, cálcio e fósforo dos ingredientes e das fezes foram realizadas de acordo com os protocolos do AOAC (1995), no laboratório de alta tecnologia Labtec em Campinas, SP, e a concentração de óxido de crômio no Laboratório de Bromatologia do DMNA da UNESP - Campus de Botucatu.

O CDa das dietas-teste dos nutrientes de cada ingrediente foram calculados de acordo com as equações propostas por FORSTER (1999).

$\mathrm{O}$ delineamento utilizado foi inteiramente casualizado com cinco tratamentos (alimentos) e quatro repetições (semanas de coleta). Os dados foram submetidos à ANOVA e quando observada diferença significativa foi aplicado o teste de comparação de médias de Tukey ao nível de $5 \%$ de probabilidade. O programa utilizado para as análises estatísticas foi o SAS (Statistical Analysis System), versão 8.12 (SAS INSTITUTE, 1985).

\section{RESULTADOS E DISCUSSÃO}

O CDa do amido e a disponibilidade aparente do Ca e P estão apresentados na Tabela 2. Entre os alimentos que apresentaram maior disponibilidade para o $\mathrm{Ca}$ e o $\mathrm{P}$ destacam-se a quirera de arroz e o sorgo, enquanto o farelo de arroz e o farelo de trigo apresentaram os menores valores de disponibilidade.

Tendência similar foi observada para o CDa do amido; entretanto, a quirera de arroz, o sorgo e o farelo de trigo apresentaram valores de digestibilidade do amido similares.

Tabela 2. Coeficiente de digestibilidade aparente do amido e disponibilidade aparente de cálcio e fósforo de ingredientes energéticos pela tilápia do Nilo $(\%, n=4$ aquários $)$

\begin{tabular}{lccccc}
\hline Nutriente & Milho & $\begin{array}{c}\text { Quirera de } \\
\text { arroz }\end{array}$ & Sorgo & Farelo de trigo & Farelo de arroz \\
\hline Cálcio & $22,18 \pm 0,77^{\mathrm{b}}$ & $43,03 \pm 1,40^{\mathrm{a}}$ & $39,89 \pm 1,42^{\mathrm{a}}$ & $5,80 \pm 0,71^{\mathrm{c}}$ & $-31,49 \pm 1,73^{\mathrm{d}}$ \\
Fósforo & $19,48 \pm 1,09^{\mathrm{c}}$ & $64,79 \pm 1,03^{\mathrm{a}}$ & $58,09 \pm 1,50^{\mathrm{b}}$ & $1,18 \pm 0,31^{\mathrm{d}}$ & $3,25 \pm 0,47^{\mathrm{d}}$ \\
Amido & $96,48 \pm 0,62^{\mathrm{b}}$ & $99,45 \pm 0,13^{\mathrm{a}}$ & $98,49 \pm 0,57^{\mathrm{a}}$ & $99,30 \pm 0,14^{\mathrm{a}}$ & $95,59 \pm 1,47^{\mathrm{b}}$ \\
\hline
\end{tabular}

Médias na mesma linha seguidas de letras diferentes são estatisticamente diferentes pelo teste de Tukey $(P<0,05)$.

FURUYA et al. (2001) encontraram valores superiores de $\mathrm{CDa}$ para o $\mathrm{Ca}$ e $\mathrm{P}$, respectivamente, para o milho $(79,50 \%$ e $45,08 \%)$ e o farelo de trigo $(76,15 \%$ e $29,51 \%)$ quando trabalharam com a tilápia utilizando dietas peletizadas. Os autores ainda afirmaram que a menor disponibilidade de minerais do farelo de trigo pode estar relacionada com o nível de fibra bruta que pode interferir na taxa de passagem e absorção dos mesmos e/ou a presença de $\mathrm{P}$ na forma de fitato. O mesmo relatam PASCOAL et al. (2006), que afirmaram haver correlação negativa entre a fibra bruta e a digestibilidade dos nutrientes. 
Já os valores de disponibilidade do $\mathrm{Ca}$ observados neste estudo para o farelo de trigo foram similares àqueles obtidos por SUGIURA et al. (1998) com o salmão $(4,5 \%)$ e a truta arco-íris $(5,4 \%)$, quando trabalharam com dietas extrusadas.

Ressalta-se ainda o valor negativo de disponibilidade do $\mathrm{Ca}$ obtido para o farelo de arroz. Esse fato tem sido descrito pela literatura em relação à disponibilidade de alguns minerais, principalmente o $\mathrm{Ca}$, para peixes. $\mathrm{O}$ baixo coeficiente de disponibilidade do Ca deve-se ao fato desse mineral encontrar-se em baixa quantidade nos ingredientes de origem vegetal e, dependendo das perdas endógenas ou da presença de fitatos, fibras e outros ligantes nesses ingredientes, a quantidade de $\mathrm{Ca}$ observada nas fezes pode ser maior que a ingestão/absorção (CHAMPAGNE, 1989). SUGIURA et al. (1998) salientaram ainda que a baixa disponibilidade do $\mathrm{Ca}$, diferente de outros minerais, pode ser devido à estrita regulação da absorção, ou mesmo pelo Ca precipitar-se no lúmen intestinal sob a forma de fosfato de $\mathrm{Ca}$, impedindo sua absorção.

Deve-se salientar que os peixes possuem alta capacidade de regular a absorção de alguns minerais (principalmente o $\mathrm{Ca}$ ) através das brânquias, podendo acarretar em $\mathrm{CDa}$ negativo devido à baixa absorção pelo trato gastrintestinal, tornando a taxa de excreção maior que a absorção.

Os valores de disponibilidade do $\mathrm{P}$ observados neste estudo são inferiores aos reportados por SUGIURA et al. (1998) com o salmão e a truta arcoíris. Esses autores obtiveram valores entre 4,01 e $55,3 \%$ para o farelo de trigo.

Essas diferenças entre estudos podem estar relacionadas a diferentes fatores. Entre eles ressaltam-se as diferenças anatomofisiológicas entre as espécies, o método de coleta de fezes empregado e o processamento utilizado para a confecção das dietas. Apesar de as dietas terem sido extrusadas, parece que o processamento não influenciou na disponibilidade do fósforo no farelo de arroz e farelo de trigo, visto que esses ingredientes apresentam boa parte do seu conteúdo em $\mathrm{P}$ na forma de fitatos. CHEFTEL (1986) ressalta que, apesar de alguns estudos demonstrarem uma redução entre $15-35 \%$ no conteúdo de fitatos de alimentos extrusados, os estudos que consideram o efeito da extrusão sobre a disponibilidade de minerais ainda encontram-se escassos e contraditórios.

Os CDa's do amido obtidos neste estudo apresentaram-se elevados para todos os ingredientes, sendo a quirera de arroz, o sorgo e o farelo de trigo os ingredientes que apresentaram maior digestibilidade.

LUPATSCH et al. (1997) obtiveram valores inferiores $(77,0 \%)$ de digestibilidade para a fração carboidrato do trigo integral com o gilthead seabream. Entretanto, a comparação de dados de outros estudos com a presente pesquisa fica difícil pelo fato de a maioria dos estudos calcular a digestibilidade de carboidratos totais, ou mesmo considerar a fração extrativo não-nitrogenado como carboidrato, enquanto nesta pesquisa foi realizada a quantificação do amido.

A menor digestibilidade do amido observada para o farelo de arroz e o milho pode estar relacionada com a composição do amido (proporção entre amilose e amilopectina) desses alimentos e uma possível formação de complexos cristalinos resistentes à hidrólise entre a fração amilose do amido e lipídeos polares, durante o processo de extrusão (CHEFTEL, 1986).

$\mathrm{O}$ alto $\mathrm{CDa}$ do amido observado neste estudo confirma a grande capacidade da tilápia do Nilo de utilizar carboidratos nas formas mais complexas e de tolerar dietas com elevados níveis de carboidratos (DEGANI \& REVACH, 1991).

\section{CONCLUSÕES}

A quirera de arroz e o sorgo apresentam alta disponibilidade de $\mathrm{Ca}$ e $\mathrm{P}$ para a tilápia do Nilo e e esta espécie é capaz de utilizar eficientemente o amido presente nos ingredientes energéticos. Estudos são necessários para elucidar o efeito do processamento na disponibilidade de minerais dos ingredientes e, desta forma, reduzir sua excreção para o meio.

\section{REFERÊNCIAS}

AOAC. Agriculture Chemicals: Contaminants, Drugs. 16.ed. Washington: Official Methods of analysis of AOAC International. 1995.

BREMER NETO, H., GRANER, C.A.F., PEZZATO, L.E., PADOVANI, C.R. Determinação de rotina do crômio em fezes, como marcador biológico, pelo método espectrofotométrico ajustado da 1,5-difenilcarbazida. Ciência Rural, v. 25, n. 3, p.691-697, 2005.

CHAMPAGNE, E.T. Low gastric hydrochloric acid secretion and mineral bioavailability. In: DINTZIS, F.R.; LASZLO, J.A. (Eds.) Mineral absorption in the monogastric GI tract. New York: Plenum, 1989. p.173184.

CHEFTEL, J.C. Nutritional effects of extrusion-cooking. Food Chemistry, v. 20, p. 263-283, 1986.

CHENG, Z.J.; HARDY, R.W. Effects of extrusion processing of feed ingredients on apparent digestibility coefficients of nutrients for rainbow trout (Oncorhynchus mykiss). Aquaculture nutrition.v, 9, p.77-83, 2003. 
CHO, C.Y.; BUREAU, D.P. A review of diet formulation strategies and feeding systems to reduce excretory and feed wastes in aquaculture. Aquaculture, v. 32, p.349360, 2001.

DEGANI, G.; REVACH, A. Digestive capabilities of three commensal fish species: carp, Cyprinus carpio L., Tilápia, Oreochromis aureus x O. niloticus, and African catfish, Clarias gariepinus (Burchell, 1882) Aquaculture and Fisheries Management, v. 22, p.397-403, 1991.

FAGBENRO, O.A. Apparent digestibility of various oilseed cakes/meals in African catfish diets. Aquaculture International, v. 6, p. 317-322, 1998.

FORSTER, I. A note on the method of calculating digestibility coefficients of nutrients provided by single ingredients to feeds of aquatic animals. Aquaculture Nutrition (Short Communication), v. 5, p.143-145, 1999.

FURUYA, W. M.; PEZZATO, L. E.; MIRANDA, E. C.; FURUYA, V. R. B.; BARROS, M. M. Coeficientes de digestibilidade aparente da energia e nutrientes de alguns ingredientes pela tilápia-do-nilo, Oreochromis niloticus (L.) (linhagem tailandesa). Acta Scientiarum, v. 23, n. 2, p.465-469, 2001.

HUA, K.; DE LANGE, C.F.M.; NIIMI, A.J.; COLE, G.; MOCCIA, R.D.; FAN, M.Z.; BUREAU, D.P. A factorial model to predict phosphorus waste output of rainbow trout (Oncorhynchus mykiss). Aquaculture Research, v.39, p.1059-1068, 2008.

LUPATSCH, I.; KISSIL, G.WM.; SKLAN, D.; PFEFFER, E. Apparent digestibility coefficients of feed ingredients and their predictability in compound diets for gilthead seabream, Sparus aurata L. Aquaculture Nutrition, v. 3, p. 81-89, 1997.

MACMILLAN, J.R.; HUDDLESTON, T.; WOOLEY,
M.; FOTHERGILL, K. Best management practice development to minimize environmental impact from large flow-through trout farms. Aquaculture, v. 226, p. 91-99, 2003.

MCGOOGAN, B.B.; REIGH, R.C. Apparent digestibility of selected ingredients in red drum (Sciaenops ocellatus) diets. Aquaculture. v. 141, p. 233-244, 1996.

PASCOAL, L. A. F.; MIRANDA, E. C.; SILVA FILHO, F. P. O uso de ingredientes alternativos em dietas para peixes. Revista Eletrônica Nutritime, v.3, n. 1, p. 292303, 2006 (acesso em 13/04/2011. Disponível em: http://www.nutritime.com.br/arquivos_internos/artigos/02 9V3N1P287_298_JAN2006.pdf

SHIAU, S.Y. Utilization of carbohydrates in warmwater fish - with particular reference to tilapia, Oreochromis niloticus $\times$ O. aureus. Aquaculture, v. 151, p. 79-96, 1997.

SUGIURA, S.H.; DONG, F.M.; RATHBONE, C.K.; HARDY, R.W. Apparent protein digestibility and mineral availabilities in various feed ingredients for salmonid feeds. Aquaculture, v. 159, p.177-202, 1998.

SUGIURA, S.H.; MARCHANT, D.D.; KELSEY, K.; WIGINS, T.; FERRARIS, R.P. Effluent profile of commercially used low-phosphorus fish feeds. Environmental Pollution, v. 140, p.95-101, 2006.

TACON, A.G.J.; FORSTERS, I.P. Aquafeeds and the environment: policy implications. Aquaculture, v. 226, p.181-189, 2003.

TAKEUCHI, T.T.; HERNÁNDEZ, M.; WATANABE, T. Nutritive value of gelatinized corn meal as a carbohydrate source to grass carp and hibrid tilapia. Fisheries Science, v. 50, n.5, p.573-577, 1994. 